Abstract In recent years, unconventional metamaterial properties have triggered a revolution of electromagnetic research which has unveiled novel scenarios of wave-matter interaction. A very small dielectric permittivity is a leading example of such unusual features, since it produces an exotic static-like regime where the electromagnetic field is spatially slowly-varying over a physically large region. The so-called epsilon-near-zero metamaterials thus offer an ideal platform where to manipulate the inner details of the "stretched" field. Here we theoretically prove that a standard nonlinearity is able to operate such a manipulation to the point that even a thin slab produces a dramatic nonlinear pulse transformation, if the dielectric permittivity is very small within the field bandwidth. The predicted non-resonant releasing of full nonlinear coupling produced by the epsilon-near-zero condition does not resort to any field enhancement mechanisms and opens novel routes to exploiting matter nonlinearity for steering the radiation by means of ultra-compact structures.

\title{
Enhanced nonlinear effects in pulse propagation through epsilon-near-zero media
}

\author{
Alessandro Ciattoni ${ }^{1, *}$ and Carlo Rizza ${ }^{2,1}$ and Andrea Marini ${ }^{3}$ and Andrea Di Falco ${ }^{4}$ and \\ Daniele Faccio ${ }^{5}$ Michael Scalora ${ }^{6}$
}

\section{Introduction}

During the last decade the metamaterial route for achieving unusual electromagnetic properties has attracted a great deal of interest in both theoretical and applied research, since it has brought to light novel electromagnetic regimes [1-6], and has suggested a number of remarkable devices for extreme manipulation of the radiation [7-9]. Structures exhibiting very small dielectric permittivity, or epsilonnear-zero (ENZ) metamaterials [10-15], belong to the family of media able to affect electromagnetic radiation in a very unconventional way because the medium's effective wavelength is much larger than the vacuum wavelength, and because they host a regime where both field amplitude and phase are slowly-varying over relatively large portions of the bulk, which is quite opposite to geometrical optics. Such key feature has been exploited to conceive setups where ultra-narrow ENZ channels are able to "squeeze" electromagnetic waves at will [16-19], and to develop new paradigms of devices for tailoring the antenna radiation pattern [20-22]. In addition, ENZ metamaterials have also been shown to support a rich phenomenology of surface waves [23-29], to achieve perfect absorption [30], to enhance spatial dispersion effects [31], and to support novel cloaking mechanisms $[32,33]$.

Other interesting mechanisms and effects arise when the ENZ regime is combined with matter nonlinearity. A kind of crucial interplay between small linear permittivity and optical nonlinearity has been identified in Ref. [34], where the authors discuss an all-optical transition from dielectric to metal behavior in a nonlinear ENZ slab. A similar mechanism has been exploited to predict a class of solitons [35] where the intensity-driven dielectric-metal transition occurs along the transverse soliton profile, yielding exotic features like transverse power flow reversing [36], unusual shapes like hollow-core [37], and two-peaked and flat-top profiles [38]. Furthermore, the combination of the ENZ regime with nonlinearity benefits from the nonresonant enhancement of the normal electric field component across the vacuum-ENZ medium interface [39], producing intriguing effects like transmissivity directional hysteresis $[40,41]$ and enhancement of second and third harmonic generation [42-45]. A different field enhancement mechanism has been identified within narrow ENZ plasmonic channels, which has been exploited to boost optical nonlinearities [46], to investigate temporal soliton excitation [47], and for the enhancement of second-harmonic generation efficiency [48]. Recently the existence of frozen light in ENZ media with cubic nonlinearity has been theoretically predicted [49].

In this work we theoretically prove that the interplay between the ENZ condition and the optical nonlinearity triggers a novel nonlinear matter-wave coupling which literally unlocks the full potential of the generally weak matter nonlinear response. By theoretically investigating the scattering of electromagnetic pulses by a thin nonlinear dispersive slab we show that a marked nonlinear pulse dynamics occurs only if the absolute value of the dielectric permittivity is very small over the pulse bandwidth. Such a nonlinear scenario stems from the fact that, in the ENZ regime, the field is spatially slowly varying and accordingly not characterized by a large number of "nodes" around which its amplitude is small, as the medium nonlinearity is allowed

\footnotetext{
${ }^{1}$ Consiglio Nazionale delle Ricerche, CNR-SPIN, Via Vetoio 10, 67100 L'Aquila, Italy ${ }^{2}$ Dipartimento di Scienza e Alta Tecnologia, Università dell'Insubria, Via Valleggio 11, 22100 Como, Italy ${ }^{3}$ ICFO-Institut de Ciencies Fotoniques, The Barcelona Institute of Science and Technology, 08860 Castelldefels (Barcelona), Spain ${ }^{4}$ SUPA, School of Physics and Astronomy, University of St. Andrews, North Haugh, St. Andrews KY16 9SS, United Kingdom ${ }^{5}$ School of Engineering and Physical Sciences, SUPA, Heriot-Watt University, Edinburgh EH14 4AS, United Kingdom ${ }^{6}$ Charles M. Bowden Research Center RDMR-WDS-WO, RDECOM, Redstone Arsenal, Alabama 35898-5000, USA

* Corresponding author: e-mail: alessandro.ciattoni@aquila.infn.it
} 

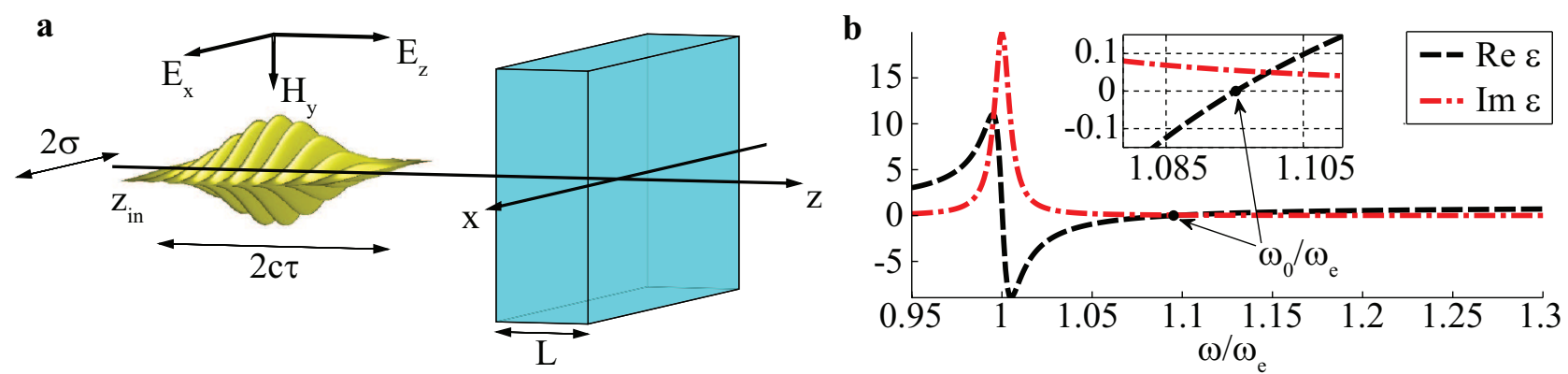

Figure 1 Pulse scattering by a slab supporting the ENZ regime. (a) Geometry of the scattering interaction between a transverse magnetic pulse which is both spatially and temporally localized and a material slab having, in the linear regime, zero-crossing-points of the real part of its dielectric permittivity. (b) In the linear regime where the medium polarization $|\mathbf{P}|$ is much smaller than the saturation polarization $P_{S}$ (see Eq.(1)), the slab has a dielectric permittivity $\varepsilon(\omega)$ (see Eq.(2)) with a standard Lorentz profile located at the resonant frequency $\omega_{e}$ and with a zero-crossing-point of its real part at $\omega_{0}$ (see Eq.(3)). Dispersion parameters have been chosen in such a way that the imaginary part of the permittivity is low around zero-crossing-point so that $|\varepsilon(\omega)|$ is much smaller than one in a spectral bandwidth around $\omega_{0}$ and the slab can support the ENZ regime.

to uniformly affect the field over the entire bulk. In other words, as a viable strategy to attain a highly nonlinear response, here we suggest to enlarge the physical volume over which the nonlinearity is effective by means of the ENZ condition, as opposed to standard approaches that resort to field enhancement mechanisms or giant nonlinear parameters.

\section{Model}

The ENZ regime occurs when $|\varepsilon(\omega)| \ll 1$ holds over the entire spectral bandwidth of the incident field. In conjunction with low absorption, this occurs if the field has a relatively narrow-band whose frequencies are very close to a zero of the real part of the medium permittivity. Permittivity zero-crossing-points are present in standard materials close to the resonant absorption frequencies and have been used to achieve the ENZ regime [42]. On the other hand, metamaterials offer an ideal platform for achieving zerocrossing-points of the permittivity since, due to the fact that the size of the unit cell is much smaller than the radiation wavelength, the effective permittivity experienced by the radiation is spatially homogeneous and its features can be tailored by choosing the relative metal-dielectric content of the inclusions [11-15]. For large optical intensities, the nonlinear response of both standard media [50] and metamaterials (in the homogeneous effective medium description) [51-53] is usually described by resorting to the paradigmatic Kerr model where the medium polarization $\mathbf{P}$ satisfies an anharmonic oscillator equation forced by the optical field $\mathbf{E}$ [50]. Here we model the dynamics of $\mathbf{P}$ through the equation [54-58]

$$
\frac{\partial^{2} \mathbf{P}}{\partial t^{2}}+\delta_{e} \omega_{e} \frac{\partial \mathbf{P}}{\partial t}+\omega_{e}^{2}\left(1+\frac{|\mathbf{P}|^{2}}{P_{s}^{2}}\right)^{-3 / 2} \mathbf{P}=\varepsilon_{0}\left(\varepsilon_{s}-1\right) \omega_{e}^{2} \mathbf{E}
$$

where $P_{s}$ is the saturation polarization that governs nonlinear oscillator behavior. This model is justified by the fact that, for $|\mathbf{P}|$ much smaller than $P_{s}$, Eq.(1) reproduces the standard Kerr anharmonic equation and for larger $|\mathbf{P}|$ it accounts for physically important higher order nonlinear terms (e.g. quintic contributions and saturation) [59]. Up to the zeroth order in $|\mathbf{P}| / P_{s}$, Eq.(1) reproduces the single-pole Lorentz oscillator with resonant frequency $\omega_{e}$, loss coefficient $\delta_{e} \omega_{e}$ and static dielectric permittivity $\varepsilon_{s}$. Therefore, in the linear regime, the dielectric permittivity experienced by monochromatic $e^{-i \omega t}$ fields is

$$
\varepsilon(\omega)=1+\frac{\varepsilon_{s}-1}{1-i \delta_{e}\left(\frac{\omega}{\omega_{e}}\right)-\left(\frac{\omega}{\omega_{e}}\right)^{2}},
$$

and it admits the zero-crossing-point

$$
\omega_{0}=\frac{\omega_{e}}{\sqrt{2}}\left\{\left(\varepsilon_{s}+1-\delta_{e}^{2}\right)+\left[\left(\varepsilon_{s}+1-\delta_{e}^{2}\right)^{2}-4 \varepsilon_{s}\right]^{1 / 2}\right\}^{1 / 2}
$$

since $\operatorname{Re}\left[\varepsilon\left(\omega_{0}\right)\right]=0$. Therefore, Eq.(1) contains all the ingredients necessary to describe the realistic and very general nonlinear response of both standard media and homogenized metamaterials also encompassing the ENZ regime.

In order to investigate such a regime we consider the scattering interaction reported in Fig.1a, where an electromagnetic pulse is launched from vacuum to orthogonally impinge on the surface of a dielectric slab whose polarization is described by Eq.(1). The material dispersion parameters are $\delta_{e}=0.01$ and $\varepsilon_{s}=1.2$ so that $\omega_{0}=1.095 \omega_{e}$ and the imaginary part of the permittivity around the zero-crossingpoint is small, as reported in Fig.1b and in its inset, thus allowing the ENZ regime. We have set for the slab thickness $L=1.25 \lambda_{e}$ where $\lambda_{e}=2 \pi c / \omega_{e}$ is the resonant wavelength and therefore, since we will mainly consider pulses whose bandwidth is localized around the zero-crossingpoint, the slab thickness is comparable with the main field wavelength. The pulse is a transverse magnetic (TM) excitation whose profile at the launching plane $E_{x}\left(x, z_{i n}, t\right)=$ $E_{0} e^{-\frac{x^{2}}{\sigma^{2}}} e^{-\frac{\left(t-t_{0}\right)^{2}}{\tau^{2}}} \sin (\bar{\omega} t)$ has time shift $t_{0}=3.178 \cdot 10^{3} \omega_{e}^{-1}$ and it is both spatially and temporally localized, $\sigma=$ 
$1.25 \lambda_{e}$ and $\tau=1.059 \cdot 10^{3} \omega_{e}^{-1}$ being its transverse and temporal widths, respectively. Note that, if the ENZ medium is a homogenized metamaterial whose unit cell size $d$ is much smaller than the wavelength $\lambda_{e}\left(d \ll \lambda_{e}\right)$, both the transverse $\sigma=1.25 \lambda_{e}$ and longitudinal $c \tau=168.5 \lambda_{e}$ pulse widths are much greater than $d$ thus consistently validating the use of the homogeneous model of Eq.(1). The amplitude $E_{0}$ and the carrier frequency $\bar{\omega}$ will be varied to investigate the ENZ regime. All the situations considered below are in the quasi-monochromatic regime since the carrier frequency $\bar{\omega}$ is always comparable with $\omega_{e}$ and the pulse spectral width $\delta \omega \simeq 1 / \tau=9.442 \cdot 10^{-4} \omega_{e}$ is much smaller than $\omega_{e}$.

Electromagnetic propagation within the slab is described by Maxwell equations coupled to Eq.(1) for the polarization so that, by using the dimensionless coordinates $\mathbf{R}=\omega_{e} \mathbf{r} / c, T=\omega_{e} t$ and dimensionless fields $\mathbf{e}=\varepsilon_{0} \mathbf{E} / P_{s}$, $\mathbf{h}=\mathbf{H} /\left(c P_{s}\right), \mathbf{p}=\mathbf{P} / P_{s}$, the pulse scattering is described by the set of equations

$$
\begin{aligned}
\nabla_{\mathbf{R}} \times \mathbf{e} & =-\frac{\partial \mathbf{h}}{\partial T}, \\
\nabla_{\mathbf{R}} \times \mathbf{h} & =\frac{\partial \mathbf{e}}{\partial T}+\frac{\partial \mathbf{p}}{\partial T}, \\
\frac{\partial^{2} \mathbf{p}}{\partial T^{2}}+\delta_{e} \frac{\partial \mathbf{p}}{\partial T}+\left(1+|\mathbf{p}|^{2}\right)^{-3 / 2} \mathbf{p} & =\left(\varepsilon_{s}-1\right) \mathbf{e}
\end{aligned}
$$

within the slab, by the first two equations with $\mathbf{p}=\mathbf{0}$ outside of the slab and matching conditions of the tangential electric and magnetic field components at the slab surfaces.

The pulse scattering by the slab both in and outside of the ENZ regime was simulated by solving the above equations through a home-made finite-difference time-domain code suitable for dealing with transverse magnetic pulses $\mathbf{e}(X, Z, T)=e_{x}(X, Z, T) \mathbf{i}+e_{Z}(X, Z, T) \mathbf{k}$, $\mathbf{h}(X, Z, T)=h_{y}(X, Z, T) \mathbf{j}$. At the edges of the computational domain perpendicular to the $Z$ axis scattering boundary condition where adopted whereas at those perpendicular to the $X$ axis the vanishing of all the field components was imposed

Note that, even though the pulse is in the quasimonochromatic regime, we here do not resort to the slowlyvarying envelope approximation (SVEA), our predictions being obtained by solving the full Maxwell equations coupled to the matter polarization equation (see Eqs.(4)) We have decided to provide a full wave analysis of the pulse scattering since the SVEA can become inaccurate in the ENZ regime. In fact the derivation of the nonlinear Schrödinger equation is based on the expansion of the wavenumber

$$
k(\omega)=\frac{\omega}{c} \sqrt{\varepsilon(\omega)}=k_{0}+k_{1}(\omega-\bar{\omega})+k_{2}(\omega-\bar{\omega})^{2}+\ldots,
$$

where $k_{n}=\left.\frac{1}{n !} \frac{d^{n} k}{d \omega^{n}}\right|_{\bar{\omega}}$, which can be truncated up to the second order by exploiting the quasi-monochromatic condition $\delta \omega / \bar{\omega} \ll 1$. In the ENZ regime where $\bar{\omega}=\omega_{0}$, the magnitudes of all the coefficients $k_{n}$ with $n \geq 1$ are very large since $\sqrt{\varepsilon\left(\omega_{0}\right)}$ and its powers appear in their denom- inators and this prevents the expansion of $k(\omega)$ to be truncated with a small number of terms. From a physical point of view, the SVEA allows to describe pulses which are long enough to contain a large number of field oscillations. In the ENZ regime, the spatial variation scale of the field is comparable with $2 \pi c /\left(\omega_{0} \sqrt{\left|\varepsilon\left(\omega_{0}\right)\right|}\right)$ and it is generally so large that a quasi-monochromatic pulse is generally not sufficiently long to contain a large number of field oscillations. Therefore a description of the ENZ regime cannot be generally based on the nonlinear Schrödinger equation.

\section{Nonlinear wave-matter interaction in the ENZ regime}

In the first set of simulations we have considered various pulses with carrier frequency located at the zerocrossing-point, i.e. $\bar{\omega}=\omega_{0}$, with different amplitudes $e_{0}=$ $\varepsilon_{0} E_{0} / P_{s}$. In Fig.2a we have plotted the absolute value of the Fourier transform $e_{x}^{F}$ (arbitrary units) of the incoming pulse $e_{x}\left(0, Z_{i n}, T\right)$ superimposed to the real and imaginary parts of the permittivity. This figure clearly shows that $|\varepsilon(\omega)|$ is much smaller than one all over the field spectrum, thus proving that the interaction occurs in the ENZ regime.

In Fig. $2 b$, which contains the main result of the present paper, we report, for a number of different amplitudes $e_{0}$, the space-time profiles of the outgoing pulses. Specifically we plot the absolute values of the analytic signals $e_{x}^{S}\left(X, Z_{\text {out }}, T\right)$ and $e_{z}^{S}\left(X, Z_{\text {out }}, T\right)$ of the field components, normalized with respect to the amplitude $e_{0}$, at the exit slab surface $Z_{\text {out }}=\omega_{e} L /(2 c)$. For $e_{0}=10^{-6}$ and $e_{0}=10^{-2}$ the normalized outgoing pulses are identical. This shows that the pulse amplitudes are small enough to prevent the matter nonlinearity from influencing the scattering. By increasing the pulse amplitude, for $e_{0}=2 \cdot 10^{-1}$ and $e_{0}=4 \cdot 10^{-1}$, the normalized outgoing pulses undergo dramatic transformation. The dependence on the input intensity is a key signature of the onset of the nonlinear regime. At higher pulse intensities, the normalized outgoing pulses having amplitudes $e_{0}=1.2$ and $e_{0}=1.6$ are almost identical, so that the nonlinear dependence of the scattering process on the input intensity saturates as expected from Eq.(1).

In Fig.2c the transition from the linear regime to the nonlinear one is pictorially shown in two different and equivalent ways: (i) the analysis of the polarization of the slab, and (ii) the spectral analysis of the field dynamics within the slab. In the first row of Fig.2c we report the space-time profile of the polarization produced within the slab by some of the pulses considered in Fig.2b, by plotting the corresponding square absolute values $\left|p_{x}^{S}(X, 0, T)\right|^{2}+$ $\left|p_{z}^{S}(X, 0, T)\right|^{2}$ of the dimensionless analytic signal of the polarization at the slab middle plane $Z=0$. The relevance of such quantity stems from the fact that it is comparable with $|\mathbf{P}|^{2} / P_{s}^{2}$ which plays a key role in the linear-nonlinear transition of the wave-matter coupling (see Eq.(1)) through the nonlinear resonant frequency

$$
\bar{\omega}_{e}(\mathbf{P})=\omega_{e}\left(1+\frac{|\mathbf{P}|^{2}}{P_{s}^{2}}\right)^{-3 / 4} .
$$



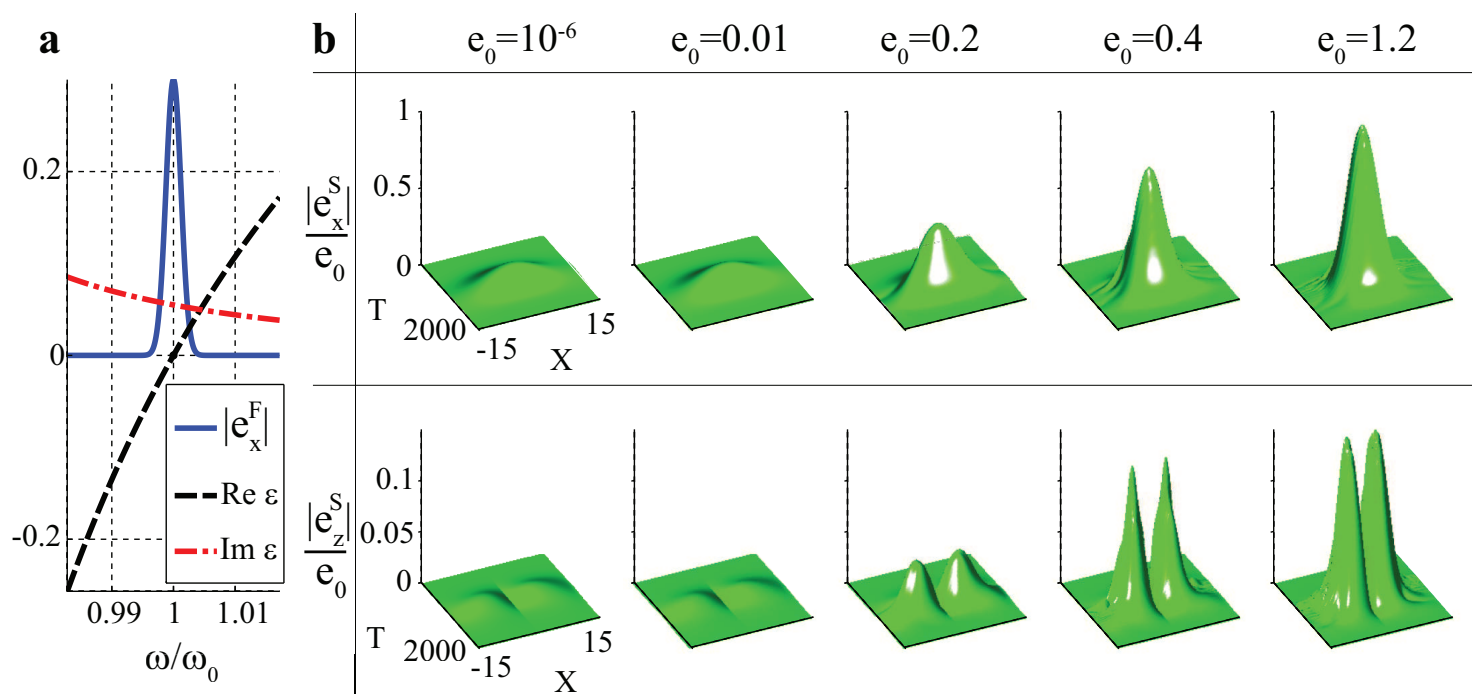

$\mathrm{e}_{0}=1.6$
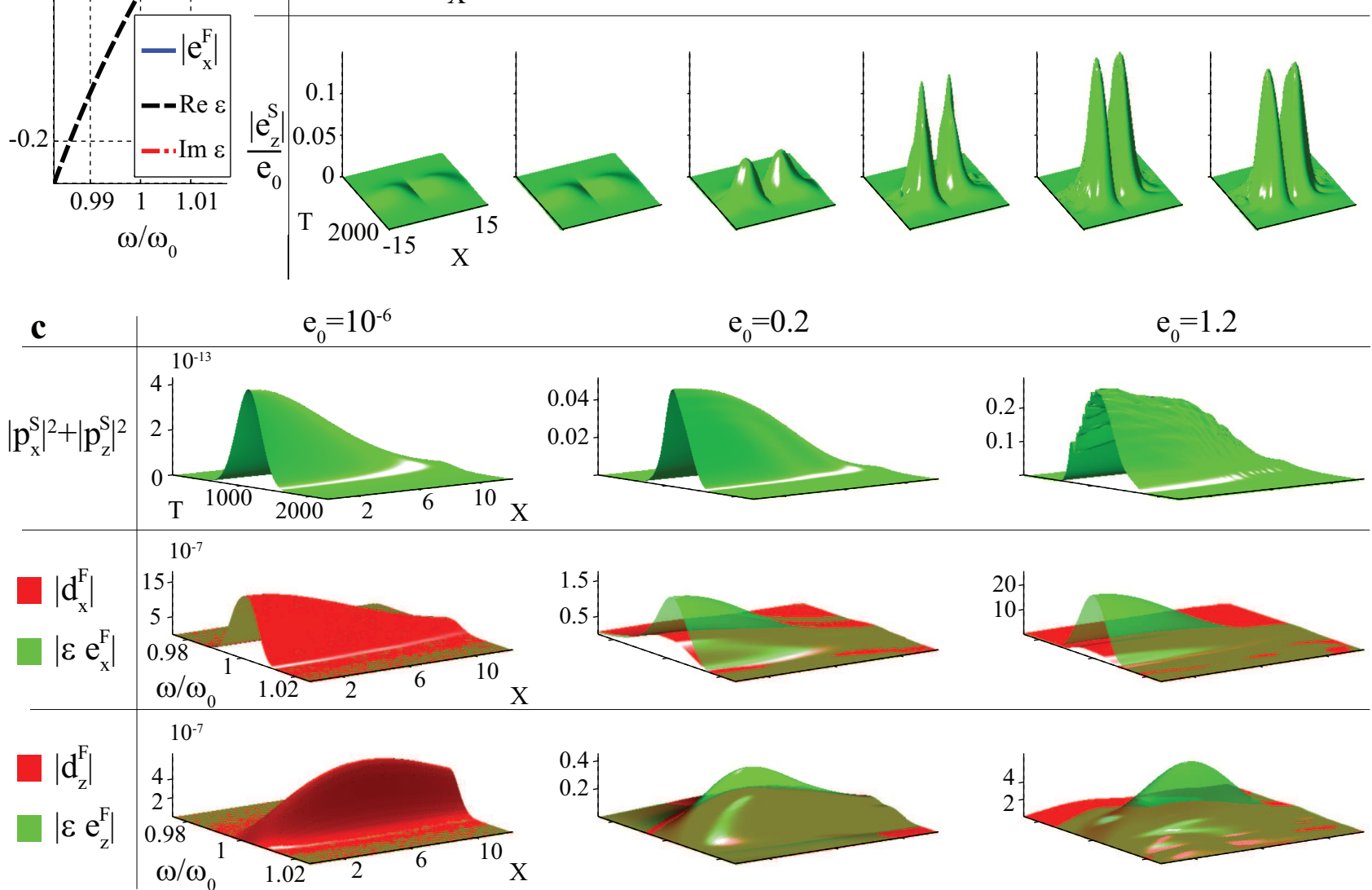

Figure 2 Nonlinear signatures of the scattering in the ENZ regime, $\bar{\omega}=\omega_{0}$. (a) Spectrum $\left|e_{x}^{F}\right|$ of the incoming pulse (arbitrary units) and real and imaginary parts of the slab linear dielectric permittivity $\varepsilon$. The interaction is in the ENZ regime since $|\varepsilon| \ll 1$ holds over the pulse spectrum. (b) Absolute values of the dimensionless analytic signals, normalized to the amplitude of the incoming pulse $e_{0},\left|e_{x}^{S}\right| / e_{0}$ and $\left|e_{z}^{S}\right| / e_{0}$ of the components of the outgoing pulse at the exit slab plane $Z_{o u t}=\omega_{e} L /(2 c)$. The dependence of $\left|e_{x}^{S}\right| / e_{0}$ and $\left|e_{z}^{S}\right| / e_{0}$ on $e_{0}$ is the key signature of the nonlinear wave-matter interaction. (c) Square absolute values of the dimensionless analytic signal of the polarization $\left|p_{x}^{S}\right|^{2}+\left|p_{z}^{S}\right|^{2}$, dimensionless displacement field components spectra $\left|d_{x}^{F}\right|$ and $\left|d_{z}^{F}\right|$ and dimensionless electric field components spectra multiplied by permittivity absolute value $\left|\varepsilon e_{x}^{F}\right|$ and $\left|\varepsilon e_{z}^{F}\right|$ at the slab middle plane $Z=0$. The occurrence of the nonlinear wave-matter interaction is further testified by two facts: 1) $\left|p_{x}^{S}\right|^{2}+\left|p_{z}^{S}\right|^{2}$ is not very much smaller than one and 2) $\left|d_{x}^{F}\right| \neq\left|\varepsilon e_{x}^{F}\right|$ and $\left|d_{z}^{F}\right| \neq\left|\varepsilon e_{z}^{F}\right|$, i.e. the standard linear constitutive relation does not hold. (In the plots where axes labels are not reported, the axes labels are those of the first plot of the row.)

For $e_{0}=10^{-6}$ the normalized square polarization is so small that $\bar{\omega}_{e}^{2}(\mathbf{P}) \simeq \omega_{e}^{2}$. Accordingly, the linear regime (where the dielectric permittivity of Eq.(2) governs electromagnetic propagation) holds. For $e_{0}=2 \cdot 10^{-1}$ the normalized square polarization is small enough to allow a perturbative description of medium nonlinearity since $\bar{\omega}_{e}^{2}(\mathbf{P}) \simeq$ $1-3|\mathbf{P}|^{2} /\left(2 P_{s}^{2}\right)$. In this case Eq.(1) is well-known to yield a nonlinear optical regime characterized by a Kerr nonlinearity [50]. For $e_{0}=1.2$ the normalized square polariza- tion is accordingly larger and does not generally allow a perturbative approximation of $\bar{\omega}_{e}^{2}(\mathbf{P})$, thus triggering the emergence of nonlinear saturation. In the second and third row of Fig.2c we plot the absolute values of the Fourier transforms $d_{x}^{F}(X, 0, \omega)$ and $d_{z}^{F}(X, 0, \omega)$ (green surfaces) of the components of the dimensionless displacement field $\mathbf{d}=\mathbf{e}+\mathbf{p}$ at the slab middle plane $Z=0$. In the same rows we also plot the absolute values $\left|\varepsilon(\omega) e_{x}^{F}(X, 0, \omega)\right|$ and $\left|\varepsilon(\omega) e_{z}^{F}(X, 0, \omega)\right|$ (red surfaces) of the Fourier trans- 


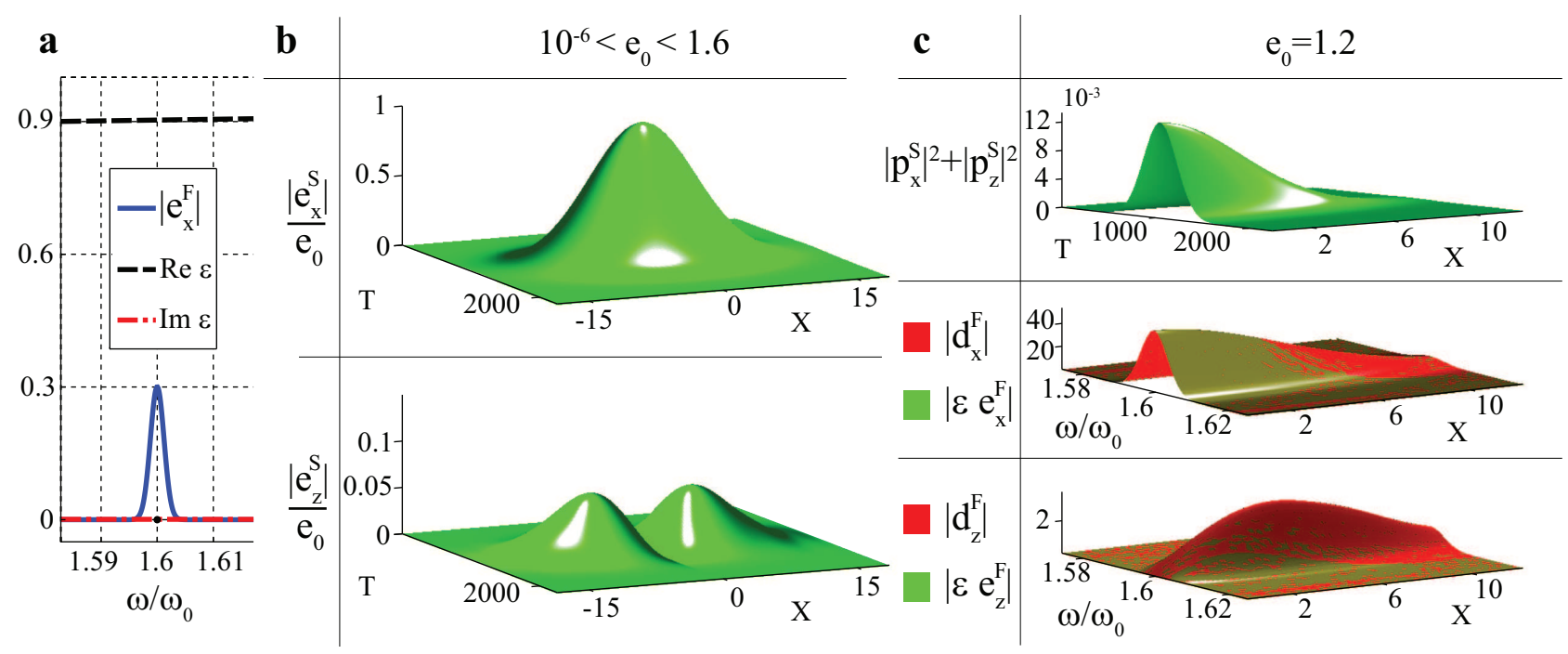

Figure 3 Linear character of the scattering outside of the ENZ regime, $\bar{\omega}=1.6 \omega_{0}$. (a) The incoming pulse spectrum $\left|e_{x}^{F}\right|$ is located at a spectral region where $|\varepsilon| \simeq 0.9$ and therefore the interaction is outside of the ENZ regime. (b) For all the incoming pulses with amplitudes in the range $10^{-6}<e_{0}<1.6$ the absolute values of the normalized analytic signals $\left|e_{x}^{S}\right| / e_{0}$ and $\left|e_{z}^{S}\right| / e_{0}$ of the outgoing pulse are effectively identical. (c) For the pulse with amplitude $e_{0}=1.2$ the square absolute values $\left|p_{x}^{S}\right|^{2}+\left|p_{z}^{S}\right|^{2}$ of the dimensionless analytic signal of the polarization within the slab are uniformly much smaller than 1 . For the same pulse amplitude the dimensionless displacement field components spectra, $\left|d_{x}^{F}\right|$ and $\left|d_{z}^{F}\right|$, coincide with the dimensionless electric field components spectra multiplied by permittivity absolute value, $\left|\varepsilon e_{x}^{F}\right|$ and $\left|\varepsilon e_{z}^{F}\right|$, within the slab. This results shows that pulse scattering outside of the ENZ regime is fully linear.

forms of the electric fields components at the same plane $Z=0$ multiplied by the linear permittivity of Eq.(2). For $e_{0}=10^{-6}$ it is evident that $\mathbf{d}^{F}=\varepsilon \mathbf{e}^{F}$ so that the standard linear relation between the displacement and the electric field holds. For $e_{0}=2 \cdot 10^{-1}$ and $e_{0}=1.2$ the discrepancy between the displacement field and its linear counterpart is dramatically evident thus restating that a marked nonlinear regime holds.

The most remarkable nonlinear trait of the scattering at hand in the ENZ regime is that the normalized amplitude of the outgoing pulse grows as the amplitude of the incident pulse is increased, as shown in Fig.2b. This phenomenology admits a simple explanation in terms of an effective nonlinear shift of the resonant frequency produced by the emergence of the nonlinear regime. From Fig.2c it is evident that the higher the amplitude $e_{0}$ of the incoming pulse the stronger the polarization induced within the slab. From Eq.(6) this produces a local decrease of the nonlinear resonant frequency $\bar{\omega}_{e}$ which can be equivalently interpreted as an effective drift of the pulse spectrum toward the spectral region where the real part of the dielectric permittivity is positive (see Fig.1b). Therefore as $e_{0}$ increases the slab becomes more transparent to the pulse and allows larger energy transmission. Since the pulse is quasi-monochromatic and its spectrum is centered at the zero-crossing-point, even a slight spectral drift produces a relatively large change of the material response, which explains the dramatic dependence of the slab transmissivity on $e_{0}$ reported in Fig. $2 \mathrm{~b}$.

It is worth noting that the interaction regime has a highly nonlinear character since a distinct pulse self-action occurs even if the slab is very thin, its thickness being comparable with the pulse carrier wavelength $\left(L=1.368 \lambda_{0}\right.$ where $\lambda_{0}=2 \pi c / \omega_{0}$ is the wavelength of the zero-crossingpoint). In addition, we observe that the described nonlinear wave-matter interaction is not due to the enhancement of the longitudinal electric field component $e_{z}$ [39-45]. Indeed, the transverse magnetic pulse incident from vacuum has a longitudinal component resulting from the finite size of the beam in the transverse direction (along the $x$-axis) and, due to the field matching at the slab edge, the longitudinal component within the slab is roughly $|\varepsilon|^{-1} \simeq 20$ times larger than its vacuum counterpart. However, from the second and third row of Fig.2c, it evident that $\left|\varepsilon e_{x}\right|$ and $\left|\varepsilon e_{z}\right|$ are comparable within the slab so that $\left|e_{x}\right|$ and $\left|e_{z}\right|$ are comparable as well. This demonstrates that in this case the longitudinal field component $e_{z}$ does not play the usual leading role we have grown accustomed to, notwithstanding the fact that the longitudinal component is still enhanced. As a consequence, the same nonlinear mechanism is triggered with incident transverse electric pulses. Therefore, a distinct advantage one gains is that, by focusing more tightly an incident transverse magnetic pulse, both enhancement of the longitudinal field and the kind of nonlinear behavior we have just discussed can in principle combine for optimal results.

In order to better appreciate the extent of the above results, it is worth estimating, in a realistic situation, the peak intensity of the pulse which triggers the linear-nonlinear transition of the scattering dynamics. Note that, in the limit 


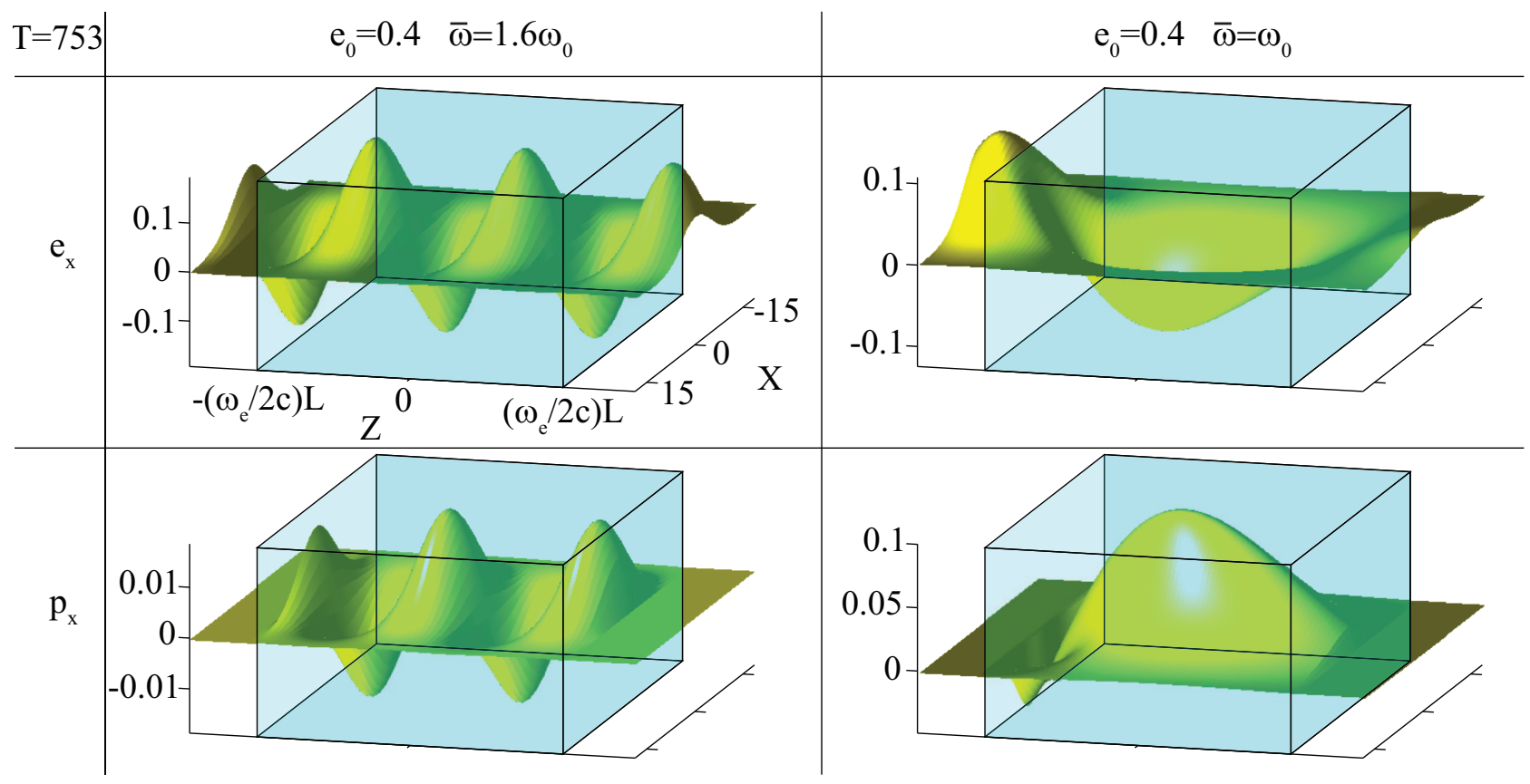

Figure 4 Mechanism supporting the full potential of the nonlinear wave-matter interaction in the ENZ regime. Spatial profiles of the dimensionless electric $e_{x}$ and polarization $p_{x}$ fields at the time $T=753$ of the pulse with $e_{0}=0.4$ and $\bar{\omega}=1.6 \omega_{0}$ (outside of the ENZ regime) and the pulse with $e_{0}=4 \cdot 10^{-1}$ and $\bar{\omega}=\omega_{0}$ (in the ENZ regime). In the first case the electric field $e_{x}$ is "large" only at the regions around the peaks of the wave and the time drift of such regions does not allow the polarization $p_{x}$ to increase and to trigger the nonlinear wave-matter coupling. In the second case, in a physically large volume, the spatially slowly varying character of $e_{x}$ yields the onset of the nonlinear regime. (In the plots where axes labels are not reported, the axes labels are those of the first plot of the array.)

\section{$|\mathbf{P}| / P_{S} \ll 1$, Eq.(1) reduces to}

$$
\frac{\partial^{2} \mathbf{P}}{\partial t^{2}}+\delta_{e} \omega_{e} \frac{\partial \mathbf{P}}{\partial t}+\omega_{e}^{2} \mathbf{P}-\frac{3 \omega_{e}^{2}}{2 P_{s}^{2}}|\mathbf{P}|^{2} \mathbf{P}=\varepsilon_{0}\left(\varepsilon_{s}-1\right) \omega_{e}^{2} \mathbf{E}
$$

which, using the well-known perturbative technique (see Ref. [50]) for extracting the third order nonlinear susceptibility $\chi^{(3)}$, yields

$$
P_{s}=\varepsilon_{0} \sqrt{\frac{3\left(\varepsilon_{s}-1\right)^{3}}{2 \chi^{(3)}}},
$$

which provides an estimation of the saturation polarization of a nonlinear medium in terms of its $\chi^{(3)}$. From panel $b$ of Fig.2, we note that the linear-nonlinear transition of the pulse scattering dynamics occurs for $e_{0} \simeq 10^{-1}$ which corresponds to $E_{0}=10^{-1} P_{S} / \varepsilon_{0}$ and to the pulse peak intensity $I_{0}=c \varepsilon_{0}\left|E_{0}\right|^{2} / 2$ given by

$$
I_{0}=10^{-2} \frac{3 c \varepsilon_{0}\left(\varepsilon_{s}-1\right)^{3}}{4 \chi^{(3)}} .
$$

For the above used value of $\varepsilon_{s}$, and for the realistic nonlinear susceptibilities $\chi^{(3)}=10^{-20}, 10^{-19}$ and $10^{-18} \mathrm{~m}^{2} / \mathrm{V}^{2}$, Eq.(9) yields $I_{0}=1.59,0.15$ and $0.01 G W / \mathrm{cm}^{2}$ which are feasible peak intensities easily achievable with picoseconds laser pulses.

\section{Absence of nonlinear effects outside of the ENZ regime}

The remarkable impact of matter nonlinearity on electromagnetic propagation in the ENZ regime is rendered even more peculiar and fascinating by the fact that no deviation from linear behavior occurs in the pulse scattering outside of the ENZ regime. This important fact is proved by a second set of simulations where we have considered various pulses identical to those discussed in Fig.2, except for their carrier frequencies, which we have set to $\bar{\omega}=1.6 \omega_{0}$. The results are reported in Fig.3, where we have plotted the same quantities considered in Fig.2. Figure 3a clearly shows that the incoming pulse spectrum $\left|e_{x}^{F}\right|$ is located in a spectral region where $|\varepsilon| \simeq 0.9$. Therefore, interaction does not occur in the ENZ regime, and the slab shows purely linear dielectric behavior. Figure $3 \mathrm{~b}$ shows that the pulse amplitude $e_{0}$ has no impact on the scattering since for all the considered pulses with $10^{-6}<e_{0}<1.6$ the profiles of the absolute values of the normalized analytic signals $\left|e_{x}^{S}\right| / e_{0}$ and $\left|e_{z}^{S}\right| / e_{0}$ are all the same. Figure $3 \mathrm{c}$ reveals that the square absolute values $\left|p_{x}^{S}\right|^{2}+\left|p_{z}^{S}\right|^{2}$ of the dimensionless analytic signal of the polarization within the slab, even for the relatively large pulse amplitude $e_{0}=1.2$, is uniformly much smaller than 1 . In addition, Fig. 3c shows that, for the same large pulse amplitude $e_{0}=1.2$, the standard linear relation $\mathbf{d}^{F}=\varepsilon \mathbf{e}^{F}$ holds between the displacement and the electric field within the slab. As a result, pulse scatter- 
ing does not reveal any nonlinear traits if the pulse carrier frequency is not close to the zero-crossing-point, no matter how large the pulse amplitude $e_{0}$ is made. We conclude that the slab governed by Eq.(1) does not play host to a nonlinear wave-matter interaction, unless the ENZ regime is exploited.

\section{Mechanism supporting the highly nonlinear wave-matter coupling in the ENZ regime}

The discussed nonlinear wave-matter interaction that occurs only in the ENZ regime admits an explanation which is closely related to the slowly varying character of the electromagnetic field imposed by the very small permittivity. In Fig. 4 we plot the spatial profiles of the dimensionless electric $e_{x}$ and polarization $p_{x}$ fields, at a specific time $T=753$, for two of the pulses considered above, and having the same amplitude $e_{0}=4 \cdot 10^{-1}$ and different carrier frequencies $\bar{\omega}=1.6 \omega_{0}$ and $\bar{\omega}=\omega_{0}$. The first pulse (left column of Fig.4) is tuned outside of the ENZ regime. Its scattering is purely linear, whereas the second pulse (right column of Fig.4) with $\bar{\omega}=\omega_{0}$ is in the ENZ regime and it has been shown to display a marked nonlinear dynamics. Consider the first pulse with $\bar{\omega}=1.6 \omega_{0}$ and note that, at the beginning of the scattering interaction, pulse propagation is evidently linear since the initial electric field is small. Therefore the profile of the pulse electric field has a considerable number of nodes, the regions of low electric field around such nodes drift in time due to propagation, the amplitude $|\mathbf{A}(\mathbf{r}, t)|$ of the electric field $\mathbf{E}(\mathbf{r}, t)=\operatorname{Re}\left[\mathbf{A}(\mathbf{r}, t) e^{-i 1.6 \omega_{0} t}\right]$ driving the polarization at each point rapidly varies between zero and it maximum value. Since in the linear regime Eq.(1) yields

$$
\mathbf{P}(t)=\varepsilon_{0} \int_{-\infty}^{t} d t^{\prime} \chi\left(t-t^{\prime}\right) \mathbf{E}\left(t^{\prime}\right),
$$

where $\chi(t)$ is the Lorentz susceptibility, we conclude that the rapid variation of the electric field amplitude forbids the polarization from locally increasing and from driving the scattering process out of the linear regime. In the case of the second pulse with $\bar{\omega}=\omega_{0}$ the initial linear dynamics forces the electric field to be spatially slowly varying within the bulk since it is in the ENZ regime. As a consequence, very few nodes appear in the electric field profile and correspondingly the amplitude $|\mathbf{A}(\mathbf{r}, t)|$ of the electric field $\mathbf{E}(\mathbf{r}, t)=\operatorname{Re}\left[\mathbf{A}(\mathbf{r}, t) e^{-i \omega_{0} t}\right]$ has a time variation scale much slower than the first pulse, occurring over a physically large portion of the bulk. Hence the polarization is efficiently pumped by the electric field (much more than the pulse tuned outside of the ENZ regime) so that it correspondingly increases to the point of triggering the onset of the nonlinear wave-matter coupling we have discussed above.

\section{Conclusion and Outlook}

In conclusion we have shown that a novel and highly nonlinear wave-matter coupling occurs if the medium's linear permittivity is very small over the entire electromagnetic field bandwidth. The realization of the full potential offered by this nonlinear matter response is due to the spatially slowly varying character of the electromagnetic field in the ENZ regime. In fact, the ENZ regime produces more efficient coupling between the electric field and the medium polarization field by enlarging the effective portion of the bulk hosting the nonlinear interaction. Unlike most strategies proposed to date in literature in order to achieve a highly nonlinear response, the nonlinear regime we have discussed here is not triggered by either cavity effects or by large nonlinear coefficients. The strategy we have outlined is simpler and more suitable to be exploited in a number a different configurations since it requires only that the field's main frequency coincides with a zero-crossing-point of the real part of the dielectric permittivity, preferably at the crossing point where absorption may be neglected. In addition, in view of such flexibility, the nonlinear coupling we have discussed may be triggered even in the presence of other mechanisms that are known to enhance matterwave coupling, thus likely to produce additional hitherto unknown, highly nonlinear effects. For example, the enhancement of the field component normal to the surface between vacuum and the ENZ material may be triggered in the scattering experiment considered in this paper simply by confining more tightly the incoming pulse along the transverse direction.

The simplicity, generality and flexibility properties of the discussed highly nonlinear regime make it an ideal platform for conceiving a number of applications and a novel generation of ultra-compact and fast devices for manipulating light. These applications would benefit of a crucial feature of the discussed regime, namely the onset on nonlinear effects at optical intensities much smaller than those commonly required in standard nonlinear optical setups. Therefore we speculate that the methodology proposed in this paper would trigger a renewed interest in nonlinear optical effects where the phenomenology is observed even with low-power laser sources.

Acknowledgements. A.C. and C.R. acknowledge support from U.S. Army International Technology Center Atlantic for financial support (Grant No. W911NF-14-1-0315). A.D.F. acknowledges support from EPSRC (EP/I004602/1). D.F. acknowledges support from the European Research Council under the European Unions Seventh Framework Programme (FP/2007-2013)/ERC GA 306559.

Key words: Nonlinear Dynamics, Nonlinear Optics, Metamaterials

\section{References}

[1] J. Valentine, S. Zhang, T. Zentgraf, E. Ulin-Avila, D.A. Genov, G. Bartal, and X. Zhang, Three-dimensional optical 
metamaterial with a negative refractive index, Nature $\mathbf{4 5 5}$, 376-379 (2008).

[2] J.B. Pendry, A chiral route to negative refraction, Science 306, 1353-1355 (2004).

[3] H. Chen, C.T. Chan, and P. Sheng, Transformation optics and metamaterials, Nature Materials 9, 387-396 (2010).

[4] A. Poddubny, I. Iorsh, P. Belov, and Y. Kivshar, Hyperbolic metamaterials, Nature Photonics 7, 948-957 (2013).

[5] A. M.Mahmoud, and N. Engheta, Wavematter interactions in epsilon-and-mu-near-zero structures, Nature Communications 5, 5638 (2014).

[6] P. Hsieh1, C. Chung, J. F. McMillan, M. Tsai, M. Lu, N. C. Panoiu, and C.W. Wong, Photon transport enhanced by transverse Anderson localization in disordered superlattices, Nature Physics 11, 268-274 (2015)

[7] J.B. Pendry, Negative refraction makes a perfect lens, Phys. Rev. Lett. 85, 3966-3969 (2000).

[8] R. Liu, C. Ji, J.J. Mock, J. Y. Chin, T.J. Cui, and D. R. Smith, Broadband Ground-Plane Cloak, Science 323, 366369 (2009).

[9] Z. Liu, H. Lee, Y. Xiong, C. Sun, and X. Zhang, Far-field optical hyperlens magnifying sub-diffraction-limited objects, Science 314, 1686-1686 (2007).

[10] N. C. Panoiu, R. M. Osgood Jr., S. Zhang, and S. R. J. Brueck, Zero-n bandgap in photonic crystal superlattices, J. Opt. Soc. Am. B 23 506-513 (2006).

[11] C. Rizza, A. Di Falco, and A. Ciattoni, Gain assisted nanocomposite multilayers with near zero permittivity modulus at visible frequencies, Appl. Phys. Lett. 99, 221107 (2011).

[12] R. Maas, J. Parsons, N. Engheta, A. and Polman, Experimental realization of an epsilon-near-zero metamaterial at visible wavelengths, Nature Photonics 7, 907-912 (2013).

[13] J. Gao, L. Sun, H. Deng, C. J. Mathai, S. Gangopadhyay, and X. Yang, Experimental realization of epsilon-near-zero metamaterial slabs with metal-dielectric multilayers, Appl. Phys. Lett. 103, 051111 (2013).

[14] L. Sun, X. Yang, and J. Gao, Loss-compensated broadband epsilon-near-zero metamaterials with gain media, Appl. Phys. Lett. 103, 201109 (2013).

[15] A. Ciattoni, R. Marinelli, C. Rizza, E. and Palange, $|\varepsilon|$-Nearzero materials in the near-infrared, Appl. Phys. B 110, 23-26 (2013).

[16] M. Silveirinha, and N. Engheta, Tunneling of Electromagnetic Energy through Subwavelength Channels and Bends using $\varepsilon$-Near-Zero Materials, Phys. Rev. Lett. 97, 157403 (2006).

[17] M. Silveirinha, and N. Engheta, Theory of supercoupling, squeezing wave energy, and field confinement in narrow channels and tight bends using $\varepsilon$ near-zero metamaterials, Phys. Rev. B 76, 245109 (2007).

[18] B. Edwards, A. Alú, M., E. Young, M. Silveirinha, and N. Engheta, Experimental Verification of Epsilon-Near-Zero Metamaterial Coupling and Energy Squeezing Using a Microwave Waveguide, Phys. Rev. Lett. 100, 033903 (2008).

[19] R. Liu, Q. Cheng, T. Hand, J. J. Mock, T. J. Cui, S. A. Cummer, and D. R. Smith, Experimental Demonstration of Electromagnetic Tunneling Through an Epsilon-Near-Zero Metamaterial at Microwave Frequencies, Phys. Rev. Lett. 100, 023903 (2008).

[20] A. Alú, M. Silveirinha, A. Salandrino, and N. Engheta, Epsilon-near-zero metamaterials and electromagnetic sources: tailoring the radiation phase pattern, Phys. Rev. B 75, 155410 (2007).

[21] J. Luo, P. Xu, and L. Gao, Directive emission based on onedimensional metal heterostructures, J. Opt. Soc. Am. B 29, 35-39 (2012).

[22] J. C. Soric, N. Engheta, S. Maci, and A. Alú, Omnidirectional Metamaterial Antennas Based on $\varepsilon$-Near-Zero Channel Matching, IEEE Trans. Antennas Propag. 61, 33-44 (2013).

[23] A. Ciattoni, C. Rizza, and E. Palange, All-optical active plasmonic devices with memory and power-switching functionalities based on $\varepsilon$-near-zero nonlinear metamaterials, Phys. Rev. A 83, 043813 (2011).

[24] S. Vassant, J. P. Hugonin, F. Marquier, and J. J. Greffet, Berreman mode and epsilon near zero mode, Opt. Express 20, 23971-23977 (2012).

[25] D. Traviss, R. Bruck, B. Mills, M. Abb, and O. L. Muskens, Ultrafast plasmonics using transparent conductive oxide hybrids in the epsilon-near-zero regime, Appl. Phys. Lett. 102, 121112 (2013).

[26] A. Ciattoni, A. Marini, C. Rizza, M. Scalora, and F. Biancalana, Polariton excitation in epsilon-near-zero slabs: Transient trapping of slow light, Phys. Rev. A 87, 053853 (2013).

[27] Y. Li, and N. Engheta, Supercoupling of surface waves with $\varepsilon$-near-zero metastructures, Phys. Rev. B 90, 201107(R) (2014).

[28] S. Campione, I. Brener, and F. Marquier, Theory of epsilonnear-zero modes in ultrathin films, Phys. Rev. B 91, 121408(R) (2015).

[29] W. D. Newman, C. L. Cortes, J. Atkinson, S. Pramanik, R. G. DeCorby, and Z. Jacob, Ferrell-Berreman Modes in Plasmonic Epsilon-near-Zero Media, Acs Photonics 2, 2-7 (2015).

[30] S. Zhong, Y. Ma, and S. He, Perfect absorption in ultrathin anisotropic $\varepsilon$-near-zero metamaterials, Appl. Phys. Lett. 105, 023504 (2014).

[31] R. J. Pollard, A. Murphy, W. R. Hendren, P. R. Evans, R. Atkinson, G. A. Wurtz, A.V. Zayats, and V. A. Podolskiy, Optical Nonlocalities and Additional Waves in EpsilonNear-Zero Metamaterials, Phys. Rev. Lett. 102, 127405 (2009).

[32] F. Bilotti, S. Tricarico, and L. Vegni, Plasmonic Metamaterial Cloaking at Optical Frequencies, IEEE Trans. Nanotechnol. 9, 55-61 (2010).

[33] E. O. Liznev, A. V. Dorofeenko, L. Huizhe, A. P. Vinogradov, and S. Zouhdi, Epsilon-near-zero material as a unique solution to three different approaches to cloaking, Appl. Phys. A 100, 321-325 (2010).

[34] A. Husakou, and J. Herrmann, Steplike Transmission of Light through a Metal-Dielectric Multilayer Structure due to an Intensity-Dependent Sign of the Effective Dielectric Constant, Phys. Rev. Lett. 99, 127402 (2007).

[35] A. Ciattoni, C. Rizza, and E. Palange, Extreme nonlinear electrodynamics in metamaterials with very small linear dielectric permittivity, Phys. Rev. A 81, 043839 (2010).

[36] A. Ciattoni, C. Rizza, and E. Palange, Transverse power flow reversing of guided waves in extreme nonlinear metamaterials, Opt. Express 18, 11911-11916 (2010).

[37] C. Rizza, A. Ciattoni, and E. Palange, Optical hollow-core waves in nonlinear Epsilon-Near-Zero metamaterials, Opt. Commun. 284, 2573-2575 (2011). 
[38] C. Rizza, A. Ciattoni, and E. Palange, Two-peaked and flattop perfect bright solitons in nonlinear metamaterials with epsilon near zero, Phys. Rev A 83, 053805 (2011).

[39] S. Campione, D. de Ceglia, M. A. Vincenti, M. Scalora, and F. Capolino, Electric field enhancement in -near-zero slabs under TM-polarized oblique incidence, Phys. Rev. B 87, 035120 (2013).

[40] A. Ciattoni, C. Rizza, and E. Palange, Transmissivity directional hysteresis of a nonlinear metamaterial slab with very small linear permittivity, Opt. Lett. 35, 2130-2132 (2010).

[41] A. Ciattoni, C. Rizza, and E. Palange, Multistability at arbitrary low optical intensities in a metal-dielectric layered structure, Opt. Express 19, 283-288 (2011).

[42] M. A. Vincenti, D. de Ceglia, A. Ciattoni, and M. Scalora, Singularity-driven second- and third-harmonic generation at $\varepsilon$-near-zero crossing points, Phys. Rev. A 84, 063826 (2011).

[43] A. Ciattoni, and E. Spinozzi, Efficient second-harmonic generation in micrometer-thick slabs with indefinite permittivity, Phys. Rew. A 85, 043806 (2012).

[44] A. Capretti, Y. Wang, N. Engheta, and L. Dal Negro, Enhanced third-harmonic generation in Si-compatible epsilonnear-zero indium tin oxide nanolayers, Opt. Lett. 40, 15001503 (2015).

[45] T. S. Luk, D. de Ceglia, S. Liu, G. A. Keeler, R. P. Prasankumar, M. A. Vincenti, M. Scalora, M. B. Sinclair, and S. Campione, Enhanced third harmonic generation from the epsilonnear-zero modes of ultrathin films, Appl. Phys. Lett. 106, 151103 (2015).

[46] C. Argyropoulos, P. Y. Chen, G. DAguanno, N. Engheta, and A. Alú, Boosting optical nonlinearities in $\varepsilon$-near-zero plasmonic channels, Phys. Rev. B 85, 045129 (2012).

[47] C. Argyropoulos, P. Y. Chen, G. DAguanno, and A. Alú, Temporal soliton excitation in an $\varepsilon$-near-zero plasmonic metamaterial, Opt. Lett. 39, 5566-5569 (2014).

[48] C. Argyropoulos, G. DAguanno, and A. Alú, Giant secondharmonic generation efficiency and ideal phase matching with a double $\varepsilon$-near-zero cross-slit metamaterial, Phys. Rev. B 89, 235401 (2014).

[49] A. Marini, and F. J. G. de Abajo, Self-organization of frozen light in near-zero-index media with cubic nonlinearity, Sci. Rep. 6, 20088 (2016).

[50] R. W. Boyd, Nonlinear Optics (Academic, San Diego, Calif., 1992).

[51] X. C. Zeng, D. J. Bergman, P. M. Hui, and D. Stroud, Effective-medium theory for weakly nonlinear composites, Phys. Rev. B 38, 10970(R) (1988).

[52] J. E. Sipe, and R. Boyd, Nonlinear susceptibility of composite optical materials in the Maxwell Garnett model, Phys. Rev. A 46, 1614 (1992).

[53] R. Boyd, and J. E. Sipe, Nonlinear optical susceptibilities of layered composite materials, J. Opt. Soc. Am. B 11, 297 (1994).

[54] J. Koga, Simulation model for the effects of nonlinear polarization on the propagation of intense pulse lasers, Opt. Let. 24, 408-410 (1999).

[55] C. Conti, A. Di Falco, and G. Assanto, Frequency generation within the forbidden band gap: All optical Rabi-like splitting in photonic crystals and microcavities, Phys. Rev. E 70, 066614 (2004).

[56] C. Conti, A. Di Falco, and, G. Assanto, Optical parametric oscillations in isotropic photonic crystals, Optics Express 12, 823-828 (2004).
[57] A. Di Falco, C. Conti, and G. Assanto, Wavelength shifting in photonic bandgap microcavities with isotropic media, Appl. Phys. Lett. 85, 4585-4587 (2004).

[58] A. Di Falco, C. Conti, and G. Assanto, Terahertz pulse generation via optical rectification in photonic crystal microcavities, Opt. Lett. 30, 1174-1176 (2005).

[59] V. Janyani, A. Vukovic, J. D. Paul, P. Sewell, and T. M. Benson, Time domain simulation in photonics: A comparison of nonlinear dispersive polarisation models, Optical and Quantum Electronics 37, 3-24 (2005). 